\title{
THE COMPARISON BETWEEN NMF AND ICA IN PIGMENT MIXTURE IDENTIFICATION OF ANCIENT CHINESE PAINTINGS
}

\author{
Yiyi Liu ${ }^{1}$, Shuqiang Lyu ${ }^{1,2,3 *}$, Miaole Hou ${ }^{1,2,3 *}$, Qinli Yin ${ }^{2}$ \\ ${ }^{1}$ School of Geomatics and Urban Spatial Informatics, Beijing University of Civil Engineering and Architecture, No.15Yongyuan \\ Road, Daxing District, Beijing, 102616-(liuyiyi, lvshuqiang, houmiaole)@bucea.edu.cn \\ ${ }^{2}$ Beijing Key Laboratory For Architectural Heritage Fine Reconstruction \& Health Monitoring, No.15Yongyuan Road, Daxing \\ District, Beijing, 102616-915172702@qq.com \\ ${ }^{3}$ Engineering Research Center of Representative Building and Architectural Heritage Database, Ministry of Education, \\ No.15Yongyuan Road, Daxing District, Beijing, 102616
}

\section{Commission III, WG III/4}

KEY WORDS: Hyperspectral Imaging, Painting Relics, Blind Source Separation Algorithm, Non-negative Matrix Factorization, Independent Component Analysis

\begin{abstract}
:
Since the colour in painting cultural relics observed by our naked eyes or hyperspectral cameras is usually a mixture of several kinds of pigments, the mixed pigments analysis will be an important subject in the field of ancient painting conservation and restoration. This paper aims to find a more effective method to confirm the types of every pure pigment from mixture on the surface of paintings. Firstly, we adopted two kinds of blind source separation algorithms, which are independent component analysis and non-negative matrix factorization, to extract the pure pigment component from mixed spectrum respectively. Moreover, we matched the separated pure spectrum with the pigments spectra library built by our team to determine the pigment type. Furthermore, three kinds of data including simulation data, mixed pigments spectral data measured in laboratory, and the spectral data of an ancient painting were chosen to evaluate the performance of the different algorithms. And the accuracy was compared between the two algorithms. Finally, the experimental results show that non-negative matrix factorization method is more suitable for endmember extraction in the field of ancient painting conservation and restoration.
\end{abstract}

\section{INTRODUCTION}

Painting cultural heritage, with brilliantly colored and vivid images, has an outstanding value in scientific study and artwork appreciation. In order to preserve these ancient artworks and show their cultural value perfectly, it's need to restore and duplication colours frequently. However, the colours observed by our naked eyes or hyperspectral cameras are usually mixtures of several kinds of pigments due to the traditional Chinese painting skills and steps. Therefore, it is necessary to determine the pigments types on surface of the paintings before restoration.

Recently, various scientific techniques, such as X-Ray diffraction, X-ray fluorescent analysis, Raman microscope analysis, electron microprobe analysis, near-infrared spectrum analysis and so on(Liu, Z. J., 2013; Zuo, J., 1999; Xia, Y., 2008 Aliatis, I., 2009), have been used in the field of conservation for cultural relics. However, most of them have some common disadvantages. Firstly, with the limitation of the instrument, environment, or other reasons, most of them need to collect samples from ancient paintings, which is a secondary damage for cultural relics. Secondly, the sampling position is mainly based on the human eyes and lacking scientific support. Finally, most of them are time consuming and can only test some small local regions of the surface. Therefore, hyperspectral technique, with the advantages of no-touching and no-sampling, is a kind of non-invasive way that has been used in the conservation study of cultural relics for decades.
Specific pigment has its specific reflective spectrum. Hence, the hyperspectral technique characterizes the nature of pigment according to the spectral information. It can be used to achieve the purpose of endmember extraction and pigment identification. The endmember extraction is necessary for obtaining spectral information of each pigment from the mixture. If we regard the mixed spectra as a result mixed by multi-source signals, the problem of extracting endmember from mixed pixels can be understood as the problem of statistical signals separation, which can be solved by using the method in the field of signal processing. The typical signal separation algorithms based on statistics are Independent Component Analysis (ICA) and Nonnegative Matrix Factorization (NMF). They have been applied in endmember extraction of hyperspectral image for many years. For example, Xiping Jiang used FastICA in separating hyperspectral mixed pixels data of real-measured rock and mine, and got the estimated value of endmember spectrum and endmember abundance(Jiang, X. P., 2013); Li Zhuo combined unsupervised endmember extraction method with blind separation algorithm, and modified the way to obtain Initial value, to consequently improve the unmixing effect of NMF applied to hyperspectral mixed pixels(Zhuo, L., 2015); according to the irrelevance between endmembers, Nan Wang used NMF, which minimized the correlation constraint between spectra, to obtain the endmember spectrum (Wang, N., 2014); Jun $\mathrm{Xu}$ optimized NMF algorithm by adding minimum convex geometry volume constraint, to realize accurate separation of

\footnotetext{
* Corresponding author. E-mail addresses: lvshuqiang@bucea.edu.cn (Shuqiang Lyu), houmiaole@ bucea.edu.cn (Miaole Hou).
} 
mixed pixels(Xu, J., 2013). However, most of these previous works are seldom related with the endmember extraction in pigments' mixtures.

In this paper, we utilized the simulation data and real-measured spectral data to compare the separation effect, the stabilization and other aspects between ICA and NMF, and the result was subsequently evaluated by using Raman spectroscopy.

\section{ENDMEMBER EXTRACTION ALGORITHMS}

Endmember extraction refers to the process of obtaining "pure" pixels from hyperspectral image, or obtaining "pure" spectra from ground measured data or spectral library. In recent years, the method based on statistical analysis for endmember extraction has drawn more and more attention (Tong, Q. X., 2016). ICA algorithm and NMF algorithm are commonly used. They were originally used to solve the problem of blind source separation, which is to separate the source signals from these observed signals exclusively. So, we adopted these two algorithms to determine the pure pigment spectrum from mixed spectra.

\subsection{Spectral Mixing Model of Pigments}

The mixing between minerals can be divided into linear mixing and non-linear mixing (Keshava, N., 2002), and mineral pigments follow the law of mineral mixing. Generally speaking, linear mixed model (LMM) has the characteristics of simple modeling, clear physical meaning and easy understanding, etc. Compared with the complex Non-linear Mixed Model (NLMM), it is simpler for computation (Chang, J. J., 2010).Many previous works (Yu, X. C., 2013; Salinas, F., 1990) show that it can be regarded as LMM when doing analysis for spectral data of mineral pigments.

The mixed spectra $X$ can be described as a linear combination of its constituent components (i.e. endmembers) with pure spectral characteristics (Zhang, J., 2004). It can be described as following:

$$
\mathrm{X}=\sum_{\mathrm{i}=1}^{\mathrm{n}} c_{i} e_{i}+\mathrm{Q}=E c+\mathrm{Q}
$$

where $\quad X=$ the mixed spectrum with a group of column vectors $\left(m^{*} 1, \mathrm{~m}\right.$ : the bands number)

$n=$ the totally endmembers number

spectrum $X$

$c_{i}=$ the proportion of the endmember $e_{i}$ in the mixed

$e_{i}=$ the endmember in the mixed spectrum $X$

$E=$ the endmembers matrix $(m * n$, which each column is vectors of end elements)

$c=$ the coefficient vector $\left(c=\left(c_{1}, c_{2}, \cdots, c_{n}\right)^{\mathrm{T}}\right)$

$Q=$ the errors

\subsection{Independent Component Analysis Algorithm}

The ICA algorithm was proposed by a French scholar named Herault in 1985(Ning, X., 2003), it was used for signal separation with blind source. Finnish scholar Hyvarinen proposed FastICA algorithm based on fixed point iterative algorithm (Sun, X. R., 1996), which can overcome the problems of traditional ICA in slow convergence and complex computation between matrices. This is done by assuming that the observed mixed pigment spectrum is linearly mixed by several endmembers' spectra, it can be expressed as:

$$
X=A s+n
$$

where $X=$ the observed signal

$A=$ the matrix of endmember spectral abundance

$S=$ the independent endmember spectrum

$n=$ the observed noise

\subsection{Non-negative Matrix Factorization Algorithm}

The NMF algorithm was firstly proposed by Daniel D. Lee and H. Sebastian Seung in 2001. This algorithm requires all elements in the matrix are non-negative. Without considering the error, the matrix is expressed as the result of the complete multiplication of two non-negative matrices (Lee, D. D., 2001). It can be expressed as follows:

$$
\mathrm{X}_{\mathrm{n} \times \mathrm{m}}=\mathrm{A}_{\mathrm{n} \times \mathrm{r}} \mathrm{S}_{\mathrm{r} \times \mathrm{m}}+\mathrm{E}_{\mathrm{n} \times \mathrm{m}}
$$

where $X=$ the mixed spectral matrix to be decomposed ( $X \in$ $\mathrm{R}_{+}{ }^{n^{\times}}$)

$A, S=$ two endmembers matrices after separation $(A \in$ $\left.\mathrm{R}_{+}{ }^{{ }^{\times}} r, S \in \mathrm{R}_{+}{ }^{r^{\times} m}\right)$

$E=$ the errors matrix, $\|E\|$ is as small as possible

$r=$ the number of endmembers

$n, m=$ is much larger than the value of $r$

\section{EXPERIMENTS AND RESULTS}

In this paper, we used three kinds of data including simulation data, mixed pigments spectral data measured in laboratory, and the spectral data of an ancient painting for mixed spectral separation. In order to give a more suitable algorithm for mixed pigments separation, we used the correlation coefficient, Root Mean Square Error (RMSE) and other indicators to judge the separation effect.

\subsection{Simulation Data Experiment}

In this experiment, we used two kinds of mineral spectra including Cinnabar and Azurite from USGS spectral database as the endmembers (as shown in (a) of Figure 1), whose coefficients meet the constraint requirement that the endmember abundance was non-negative and the sum of endmember abundance was 1 , to generate five mixed spectra with different abundances.

We took "75\% Cinnabar 25\% Azurite $+25 \%$ Cinnabar $75 \%$ Azurite" as an example, the spectra after initial separation by FastICA were shown in (b) of Figure 1. It can be seen that, there is a big difference between the spectral curves of the component ica-2 and endmember. After adjusting the components order, we got the comparison result and the correlation coefficient between the endmember and the independent component separated by FastICA, as shown in (c) and (d) of Figure 1. It can be seen that, the overall shapes of the components spectra with order adjustment are basically the same as the endmembers spectra, and also the correlation coefficient are high. Figure 2 is the comparison result and the correlation coefficient between the endmember and the independent component separated by NMF. It can be seen that the similarity of the component and endmember is high. 
In general, the NMF algorithm has the advantages of good stability, high repeatability and relatively high accuracy of the separated components, which is suitable for hyperspectral mixed pigments separation.

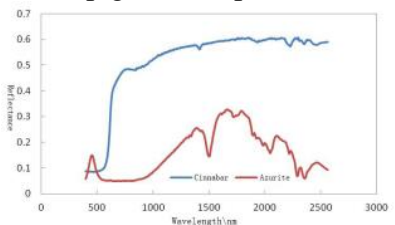

(a) the spectra of endmembers selected from USGS

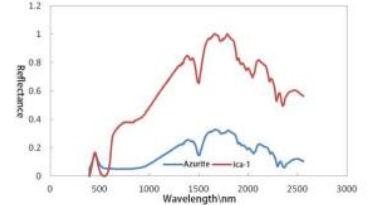

(c) the comparison between the endmember Azurite and the component1 with order adjustment (correlation coefficient: 0.99 )

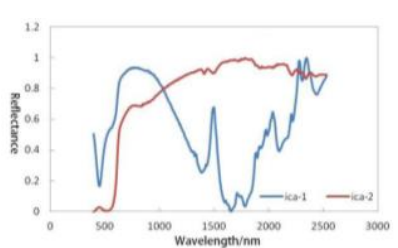

(b) the spectra after initial separation

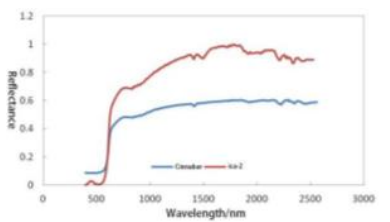

(d) the comparison between the endmember Cinnabar and the component 2 with order adjustment

(correlation coefficient: 0.84 )

Figure.1 The comparison of separation result by FastICA

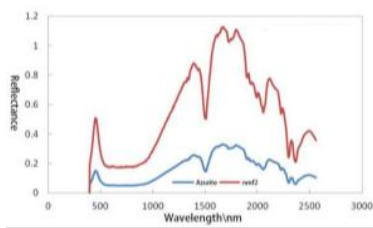

(a) the comparison between the independent component and the endmember Azurite

(correlation coefficient:0.999967)

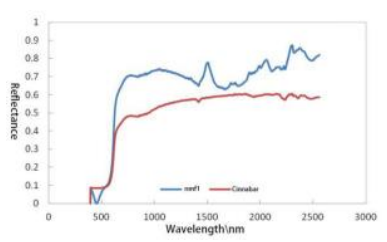

(b) the comparison between the independent component and the endmember Cinnabar

(correlation coefficient:0.9711)

Figure. 2 The comparison of separation result by NMF

\subsection{Laboratory Mixed Pigments Samples Experiment}

In order to study the mixed spectral separation effect of actual mineral pigments by two algorithms, we designed several groups of pigments samples for experiment. Each pigment group was mixed with a certain proportion (as shown in Table 3 ), and respectively obtained five mixed spectra with different abundances. We took the Zinnober-Gamboge group as an example, the separation results of mixed pigments spectra by FastICA and NMF were shown in Figure 4.

\begin{tabular}{|c|c|c|}
\hline Mixture & Zinnober/\% & Gamboge $/ \%$ \\
\hline 1 & 90 & 10 \\
2 & 75 & 25 \\
3 & 50 & 50 \\
4 & 25 & 75 \\
5 & 10 & 90 \\
\hline
\end{tabular}

Table. 3 the abundances of mixed pigments

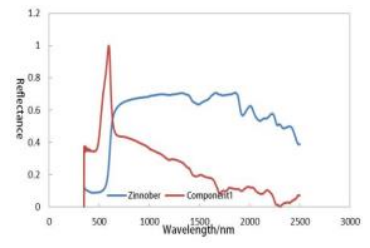

(a) the separation result of Zinnober by FastICA

(correlation coefficient: -0.334)

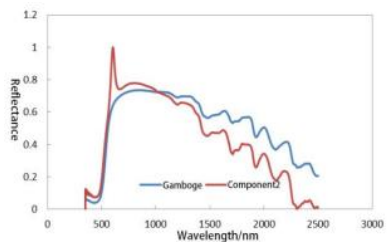

(b) the separation result of Gamboge by FastICA

(correlation coefficient: 0.905 )

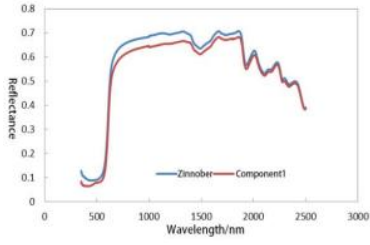

(c) the separation result of Zinnober by NMF

(correlation coefficient: 0.997) (d) the separation result of Gamboge by NMF

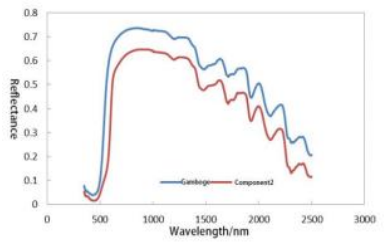

Figure.4 The separation results of the Zinnober-Gamboge group

The experimental results show that the quality of components separated by NMF is better than it by FastICA because the components spectra separated by NMF are highly similar to the endmembers spectra, and the shapes of spectral curves are basically the same, as well as the correlation coefficient is higher. On the other hand, the components separated by FastICA are not similar with the endmembers spectra, and the trend of their reflective curves is even opposite to each other.

\subsection{Real Ancient Painting Experiment}

The selected ancient painting is a landscape painting of late Qing Dynasty. The six spectra with the same pigment of ROIs on the surface of the painting were collected by ASD spectrometer. The specific selected points were shown in Figure 5 .

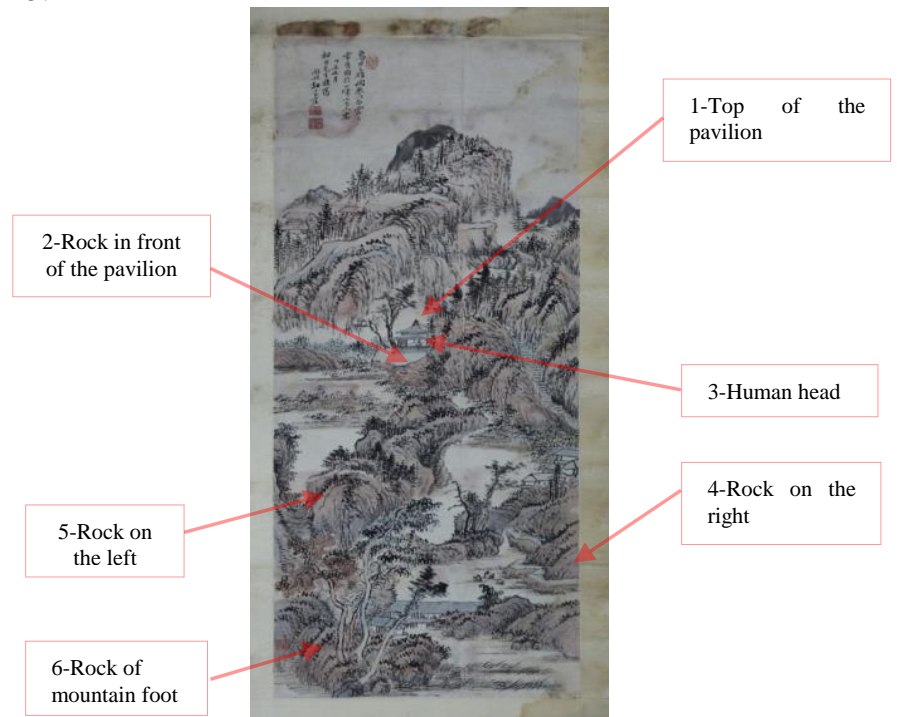

Figure.5 ROIs in the ancient painting

We used the spectra of two points"1-Top of the pavilion" and "2-Rock in front of the pavilion" as the spectral matrix to be separated, and respectively used FastICA and NMF for spectra separation. And matched the separated components with the spectra in the spectral library (Here we used the component matching result of the NMF). According to the top three matching results shown in Table 6, it can suggest that the best matching results of the two components $n m f 1$ and $n m f 2$ are $\mathrm{Fe}_{2} \mathrm{O}_{3}$ and lead powder.

\begin{tabular}{|c|c|c|c|}
\hline Components & Result 1 & Result 2 & Result 3 \\
\hline nmf1 & $\begin{array}{c}\text { Terracotta } \\
\left(\mathrm{Fe}_{2} \mathrm{O}_{3}\right)\end{array}$ & $\begin{array}{c}\text { Ocher } \\
\left(\mathrm{Fe}_{2} \mathrm{O}_{3}\right)\end{array}$ & $\begin{array}{c}\text { Mineral } \\
\text { yellow } \\
\left(\mathrm{As}_{2} \mathrm{~S}_{3}\right)\end{array}$ \\
\hline $\mathrm{nmf} 2$ & $\begin{array}{c}\text { Lead powder } \\
\left(\mathrm{Pb}(\mathrm{OH})_{2} .\right.\end{array}$ & Yunbai & $\begin{array}{l}\mathrm{Calcite} \\
\left(\mathrm{CaCO}_{3}\right)\end{array}$ \\
\hline
\end{tabular}




\begin{tabular}{|l|l|l|}
\hline & $\left.2 \mathrm{PbCO}_{3}\right)$ & \\
\hline
\end{tabular}

Table. 6 The matching results of NMF

In order to verify whether the matching results were correct, Raman spectroscopy was performed on the same area of the paining. The results in Figure 7 were $\mathrm{Fe}_{2} \mathrm{O}_{3}$. However, the lead powder was not detected probably due to its low content, limitations of the instrument, or other reasons.

The type of pigment contained in the area cannot be verified completely, but it can be deduced that the types of pigments are Ocher and lead powder by combining with the painting knowledge and the pigment matching result, which is consistent with the result of NMF. As a result, NMF algorithm can also have good effect on the mixed pigments separation of the actual painting, and has great application value for mixed pigments analysis.

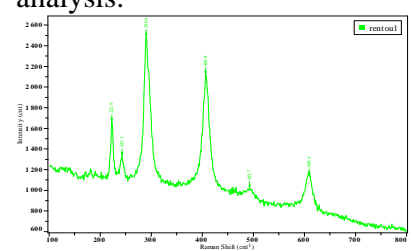

(a) Raman result of human head $\left(\mathrm{Fe}_{2} \mathrm{O}_{3}\right)$

Figure.7 The results of Raman spectroscopy

\section{CONCLUSION}

In this paper, we respectively used two kinds of blind source algorithms FastICA and NMF for mixed spectral separation to obtain several components of the basic pigments, and then to determine the pigments types by calculating the similarity between components and endmembers in pigments spectral library. Firstly, we used simulation data and mixed pigments spectral data measured in laboratory to compare the separation effect of the two algorithms. Secondly, we analysed the spectral data of an ancient painting in the late Qing Dynasty and verified the separation results by Raman Spectroscopy. Finally, the experimental results show that, compared with FastICA, NMF has higher performance both in separation quality and separation stability, and it is more suitable for spectral separation of mixed pigment.

\section{ACKNOWLEDGEMENTS}

This paper is funded by the research fund of the National key research and development program (No.2016YFB0501404, No.2017YFB1402100) and the National Natural Science Foundation of China (No. 41171304, No. 41371492).

\section{REFERENCES}

Aliatis, I., Bersani, D., Campani, E., et al., 2009. Green pigments of the Pompeian artists' palette. Spectrochimica Acta Part A Molecular \&Biomolecular Spectroscopy, 73(3), pp. 5328.

Chang, J. J., 2010. Raman Studies of the Pigments in Ancient Wall Paintings and Dyes. Journal of Jilin University.

Jiang, X. P., Wu, F. H., Jiang, Y., et al., 2013. Hyper-Spectral Mineral Abundance Inversion Based on FastICA Algorithm.
Journal of Jilin University (Earth Science Edition), 43(5), pp.1681-1686.

Keshava, N., \& Mustard, J. F., 2002. Spectral unmixing. IEEE Signal Processing, 19, pp.44-57.

Lee, D. D., Seung, H. S., 2001. Algorithm for Non-Negative Matrix Factorization. Advances in Neural Information Processing Systems. Vol. 13.

Liu, Z. J., Wang, J. Y., Han, L. G., et al., 2013. Raman Spectra of Some Mineral Pigments Used in Ancient Chinese Artworks (2).The Journal of Light Scattering, 24(2), pp. 170-175.

Ning, X., 2003. Study on Ratio Spectrum and Derivative Spectrophotometric Method in Multicomponent Analysis. Ocean University of China. pp. 20-21.

Salinas, F., Nevado, J. J., Mansilla, A. E., 1990. A new spectrophotometric method for quantitative multicomponent analysis resolution of mixtures of salicylic and salicyluric acids. Talanta, 37(3), pp. 347-351.

Sun, X. R., Lin, Z. D., Zhang, J. Y., 1996. Discrimination of Color Difference of Surface. Acta Psychologica Sinica, 28(1), pp.9-15.

Tong, Q. X., Zhang, B., Zhang, L. F., 2016. Current progress of hyperspectral remote sensing in China. Journal of Remote Sensing, 20(5), pp.689-707.

Wang, N., Zhang, L. P., Du, B., 2014. Minimum Spectral Correlation Constraint Algorithm Based on Non-negative Matrix Factorization for Hyperspectral Unmixing. Geomatics and Information Science of Wuhan University, 39(1), pp. 22-26.

Xia, Y., Wu, S.C., Cui, S. K., et al., 2008. Study on the Pigments in Polychrome Potteries of West Han Dynasty Tomb in Weishan, Shandong Province. Sciences of Conservation and Archaeology, 20(2), pp. 13-19.

Xu, J., Zhan, A. Y., Liu, Z. W., 2013. The Spectral Unmixing Based on OSP and NMF. Journal of East China Jiaotong University, 2013(1), pp.5-9.

Yu, X. C., 2013. Hyperspectral Image Analysis and Application. Science Press.

Zhang, J., Rivard, B., Sanchez-Azofeifa, A., 2004. Derivative spectral unmixing of hyperspectral data applied to mixtures of lichen and rock. Ieee T Geosci Remote, 42(9), pp. 1934-1940.

Zhuo, L., Cao, J. J., Wang, F., et al., 2015. Blind unmixing based on improved target endmember for hyperspectral imagery. Journal of Remote Sensing, 19(2), pp. 273-287.

Zuo, J., Xu, C.Y., 1999. The Study of Ancient Coating Pottery and Wall Painting by Raman Spectra. The Journal of Light Scattering, 1999(3), pp. 215-219. 